\title{
efectos del árido sobre ciertas propiedades del hormigón de cemento portland
}

SANDOR POPOVICS

Profesor de la Universidad de Auburn, Alabama, U.S. A.

sinopsis

$659-1$
Este trabajo da una descripción concisa de los efectos que tienen las propiedades princi. pales de los áridos sobre el comportamiento del hormigón de cemento portland endurecido. Se discuten los efectos de la resistencia, elasticidad, composición química, tamaño y super. Se discuten los efectos de la resistencia, elasticidad, composición quimica, tamaño y super-
ficie de los áridos. Las propiedades del hormigón endurecido que se discuten incluyen la resistencia a compresión y flexión, contracción, resistencia al roce y al resbalamiento.

\section{Resistencias a la compresión}

Dado que aproximadamente las tres cuartas partes del volumen del hormigón están constituidas por árido o áridos minerales, es evidente que las propiedades del árido influyen en la calidad del hormigón. Propiedades tales como la resistencia mecánica, la elasticidad, el tamaño y la textura superficial de las partículas de árido, así como el tipo y cantidad de materiales nocivos, producen un efecto directo. También la granulometría y la forma de las partículas, a través del requerimiento de agua y de la trabajabilidad de la mezcla, ejercen una acción indirecta sobre la resistencia del hormigón. Estos efectos se hacen más importantes a medida que la resistencia del hormigón crece. La incompatibilidad de tipo térmico, o de cualquier otro tipo, también influye desfavorablemente sobre la resistencia del hormigón. Además, cualquier reacción química entre el árido y la pasta de cemento puede ocasionar un aumento o una disminución de la resistencia del hormigón. En lo que sigue se describen algunos de estos efectos.

Por lo que respecta al efecto de la resistencia del árido en la resistencia del hormigón, ciertos datos experimentales demuestran que la resistencia a la compresión del hormigón parece disminuir cuando el tanto por ciento de desgaste de los áridos determinado mediante el ensayo de Los Angeles aumenta, siempre que la resistencia del árido no exceda considerablemente de la resistencia del hormigón (101), (102) y (103). En casos de gran resistencia del árido no es evidente la existencia de una relación entre el resultado del ensayo de Los Angeles y la resistencia a la compresión del hormigón (104) y (105). Debe indicarse que en otros países se han normalizado diversos métodos de ensayo para la determinación de la resistencia de los áridos a la compresión y al impacto. Tales son, por ejemplo, el método descrito en la norma británica B.S. 812:1960 y el método de la norma alemana DIN 52109.

La figura 1 muestra, según Walker y Bloem (106), el efecto del tamaño máximo de partícula sobre la relación entre la resistencia y el factor agua/cemento para dos contenidos de cemento diferentes. Si bien hay una dispersión considerable, puede observarse que para una relación agua/cemento y un contenido de cemento dados, se obtuvo mayor resistencia con árido de $3 / 4$ de pulg. que con árido similar de $1 \frac{1 / 2}{2}$ pulgadas.

Otras experiencias afines conducen a una conclusión semejante (107), (108), (109) y (110), especialmente para las relaciones agua/cemento más bajas. La razón probable de este fenómeno es que la fuerza unitaria de adherencia entre la pasta de cemento endurecida y el árido aumenta al disminuir el área superficial del árido grueso, es decir, al aumentar el tama- 


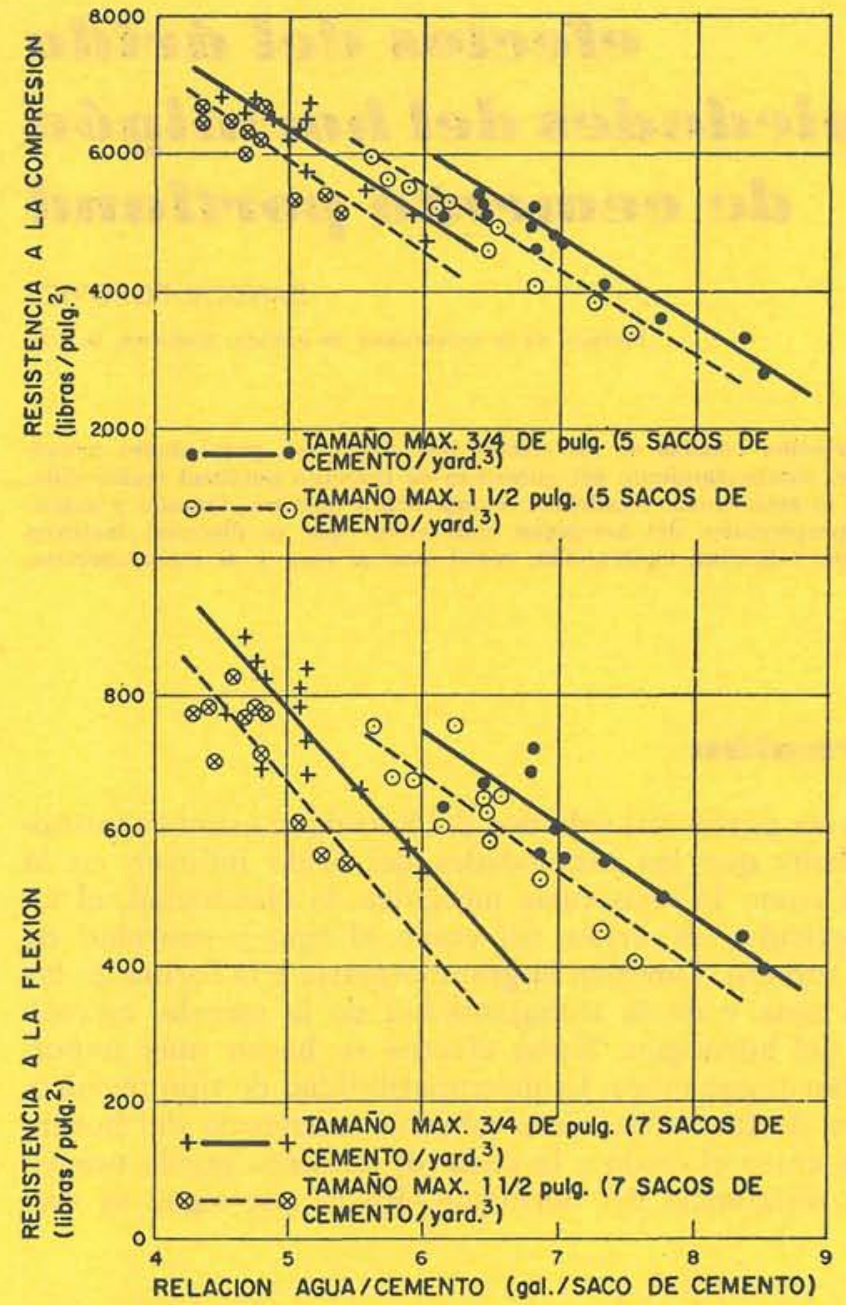
Fig. 1. Relaciones entre el factor agua/cemento y la resistencia para
dos tamaños máximos de partícula y dos contenidos de cemento (106). ño máximo de partícula. No obstante, en los casos prácticos el efecto de la variación con el tamaño máximo de partícula es menos pronunciado, a causa de que la cantidad de agua de amasado necesaria para una consistencia dada puede reducirse aumentando el tamaño máximo de partícula. Esto compensa, más o menos, la reducción de la resistencia del hormigón debida al aumento del tamaño máximo de partícula. Esta compensación es particularmente sustancial en casos de contenidos de cementos bajos (109).

Mediante una reciente serie de ensayos se ha llegado a demostrar la existencia de otra forma de compensación (111). Los resultados obtenidos muestran también el efecto combinado de la forma y textura superficial de las partículas del árido sobre la resistencia a la compresión del hormigón. En esta serie de ensayos se confeccionó hormigón con un árido grueso consistente en una grava de 1 pulg. de tamaño máximo. Se comparó la resistencia a la compresión de este hormigón con la de otros hormigones de igual consistencia, esto es, con un contenido de agua ligeramente menor, en los cuales se utilizó como árido grueso determinadas combinaciones de grava con tamaño máximo de 1 pulg. y caliza machacada con tamaño máximo de 2 pulg. Los resultados de los ensayos mostraron un aumento en la resistencia a la compresión del 5 al 10 por 100, aproximadamente, causado por la adición de piedra caliza gruesa machacada. Se apreció también que la sustitución de un árido redondeado por un material machacado produce un incremento de la resistencia aún mayor, si el tamaño máximo, la relación agua/cemento y el contenido de aire permanecen invariables (112). No obstante, si se emplea como árido fino un material de machaqueo, o la forma de las partículas es desfavorable, el uso de áridos machacados exige un gran aumento del agua de amasado para mantener la trabajabilidad adecuada, lo cual, para un contenido fijo de cemento, reduce el aumento de resistencia.

La causa de estos aumentos de resistencia parece ser que la adherencia entre la pasta de cemento y la piedra de machaqueo es mayor que la adherencia entre la misma pasta y la grava de igual tamaño. Cordon (110) y Kaplan (113) llegaron a una conclusión semejante.

El recubrimiento, es decir, una capa depositada sobre la partícula después de su formación, puede reducir la adherencia y, por lo tanto, la resistencia del hormigón para una relación agua/cemento dada. Por ejemplo, una película de arcilla que se adhiere a las partículas de árido grueso, normalmente perturba la adherencia, reduciendo así la resistencia del hormigón en un 10 e incluso un 15 por 100. 
Es sorprendentemente limitada la información disponible en la literatura técnica acerca de la influencia de la fracción extremadamente fina de áridos, tal como los materiales que pasan el tamiz número 200. Existe una unanimidad general en cuanto a que tales materiales no deben permitirse en grandes cantidades, a causa de su tendencia a aumentar la cantidad de agua exigida por la mezcla. Hay casos en los que una pequeña cantidad de un material muy fino puede mejorar la trabajabilidad; pero un exceso produce una seria reducción de la misma, particularmente en el caso del polvo de granito (114 a), probablemente a causa del contenido de mica (115 a). Sin embargo, aún no está claro qué debe entenderse por "excesivo». Existe, por ejemplo, la opinión de que el "polvo" puede ser permisible hasta un porcentaje del 20 por 100, según las circunstancias (114 b).

Graf presenta datos numéricos relativos a los efectos de varias arcillas y limos sobre la demanda de agua y la resistencia de los morteros (116 a). Encontró que cuanto más fina es la arcilla, más pronunciado es su efecto sobre las propiedades del mortero. Este efecto se pudo apreciar ya para contenidos de arcilla tan bajos como el 3 por 100; pero cuando el contenido fue del 15 por 100, la resistencia a la compresión del mortero se redujo a un tercio de su valor original. Investigaciones recientes destacan el hecho de que, no sólo la finura de la arcilla y del limo pueden influir en las propiedades del hormigón, sino también la composición mineralógica. Valdría la pena examinar si el «ensayo del equivalente de arena» (117) es adecuado para evaluar tanto la cantidad como la actividad de una arcilla con respecto a la resistencia del hormigón.

La granulometría del árido puede afectar directamente a la resistencia a la compresión del hormigón; a saber, cuanto más gruesa es la granulometría del árido dentro de los límites de una trabajabilidad adecuada, mayor es generalmente la resistencia del hormigón, suponiendo la misma relación agua/cemento, la misma proporción de mezcla y el mismo tamaño máximo de partícula. Este aumento de resistencia puede llegar a ser del 15 por 100 en determinados casos (118), (119) y (120). No obstante, debe advertirse que las experiencias de Lenhard no muestran un efecto tan directo de la granulometría (121).

El efecto directo de la granulometría en la resistencia del hormigón aparece como secundario en comparación con el efecto indirecto a través de la cantidad necesaria de agua de amasado y la trabajabilidad. A este respecto, una granulometría es óptima, cuando produce la trabajabilidad requerida con el mínimo de agua. Así, pues, granulometrías más finas que la óptima, las llamadas sobredosificadas de arena, rebajan indirectamente la resistencia del hormigón, a través del aumento de la demanda de agua, mientras que granulometrías más gruesas que la óptima, es decir, las subdosificadas de arena, reducen la resistencia a causa de la trabajabilidad perjudicada y del mayor contenido de aire resultante. Vale advertir, no obstante, que, por ejemplo, una disminución de 0,25 en el módulo de finura de un árido ya sobredosificado de arena tiene consecuencias mucho más nocivas que la misma disminución de 0,25 cuando la granulometría se aproxima a la óptima (122).

Por lo que respecta a la forma de las partículas, la principal objeción a una excesiva cantidad de partículas planas o alargadas en el árido es su efecto perjudicial para la trabajabilidad del hormigón y la consiguiente necesidad de mezclas más altamente dosificadas de arena, lo que implica el empleo de más cemento y agua (115b).

Finalmente, existe una evidencia experimental de que las mezclas con áridos saturados dan mayores resistencias al hormigón que las mismas mezclas con áridos secos (123) y (124). El efecto de la saturación del árido sobre el aumento de la resistencia es más pronunciado (10 a 15 por 100) para relaciones agua/cemento bajas, esto es, para resistencias altas. Esto indica que la humedad absorbida en las partículas del árido ayuda a la hidratación del cemento y actúa como un agente interno de curado. 
En general, es de esperar que distintos áridos gruesos den lugar a diferentes resistencias a la compresión en hormigones comparables. En una serie de ensayos llevada a cabo por Walker y Bloem (104) con 16 áridos gruesos de la misma granulometría, la desviación máxima de la resistencia a la compresión, con un árido dado, respecto de la media de todos los áridos, fue aproximadamente del 8 por 100. El valor de la desviación máxima encontrado por Kaplan en otra serie de ensayos (113) fue más o menos del doble.

\section{Resisstemciass a la fllexión}

La resistencia a la flexión de un hormigón se ve afectada principalmente por aquellas propiedades del árido que influyen sobre la adherencia entre el árido y la pasta de cemento. Tales propiedades son la textura superficial, la forma de las partículas, la limpieza de la superficie y el tamaño máximo de las partículas. Estas propiedades producen mayores efectos sobre la resistencia a la flexión de un hormigón que sobre la resistencia a la compresión. Consecuencia natural de este hecho es que la relación entre las resistencias a la flexión y a la compresión varía con el tipo de árido (125). Por ejemplo, la resistencia a la flexión de un hormigón hecho con piedra machacada como árido grueso es considerablemente mayor que la de un hormigón comparable hecho con grava. Esto es cierto, aún en el caso de man-

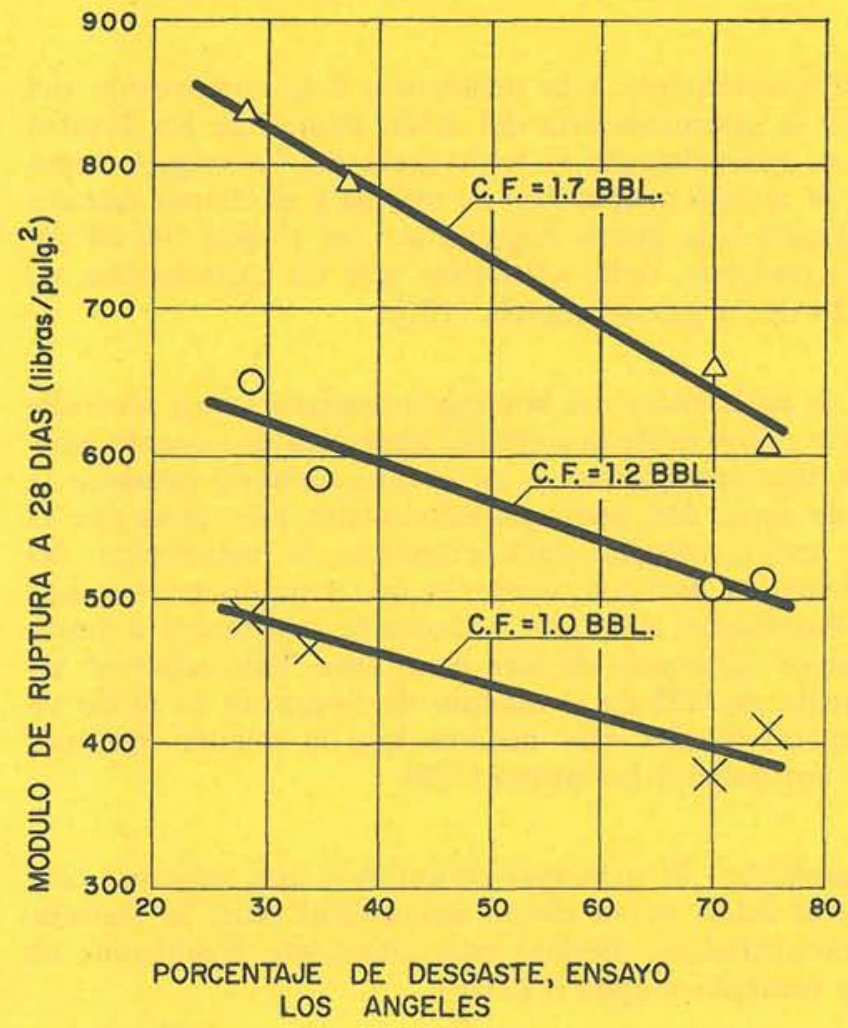

Fig. 2. Relación entre resistencia a flexión del hormigón y pérdidas por abrasión Los Angeles del árido grueso. I (126). tener fija la consistencia, en lugar de la relación agua/cemento, con tal de que el contenido de cemento permanezca invariable. De forma análoga la figura 1 muestra la reducción de la resistencia a la flexión debida al aumento del tamaño máximo de particula (106). El efecto directo de la granulometría en la resistencia a la flexión está indicado por las experiencias de Singh (119). Los efectos del árido sobre la resistencia a la flexión del hormigón son tanto más importantes cuanto mayor llega a ser la resistencia del hormigón.

Como indican las figuras 2, 3 y 4 (126) y (127), la resistencia a la flexión del hormigón parece disminuir más fuertemente que la resistencia a la compresión, al aumentar el porcentaje de desgaste del árido en el ensayo de Los Angeles. A pesar de la aparentemente buena correlación, los valores no muestran numéricamente el efecto de las pérdidas por abrasión según el ensayo de Los Angeles sobre la resistencia a la flexión del hormigón. Esto es así debido a que si dos hormigones se hacen con dos áridos gruesos distintos, pero, por otra parte, de idéntica manera, la diferencia entre las resistencias a la flexión es debida, no solamente a la diferencia entre las pérdidas por abrasión según el ensayo de Los Angeles, sino, además, a las diferencias existentes entre los tamaños y las texturas superficiales, la elasticidad, etc., de los dos áridos. 

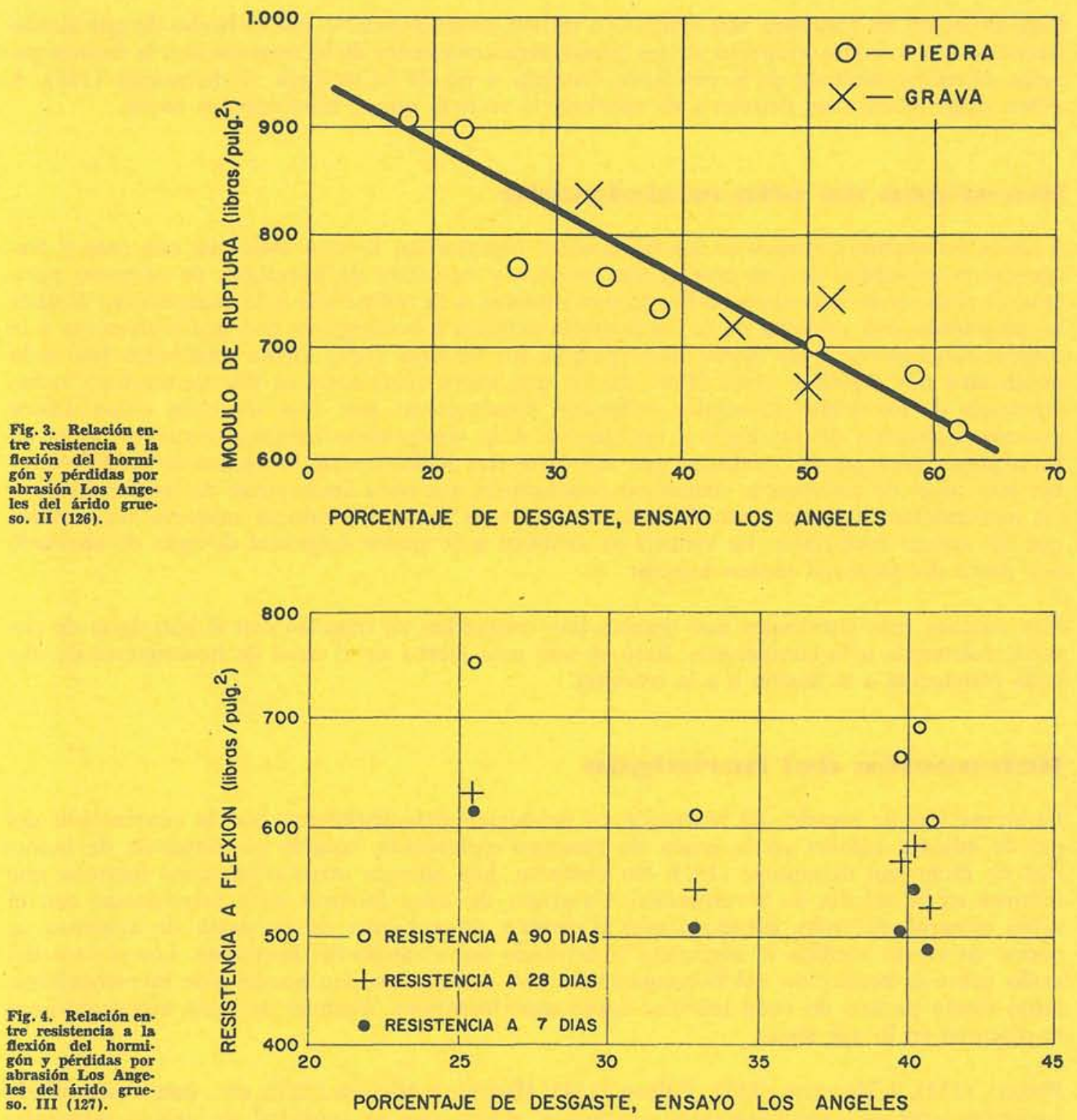

Los recubrimientos sobre la superficie de las partículas del árido grueso y una excesiva cantidad de la fracción extremadamente fina del árido reducen también la resistencia a la flexión del hormigón (116 a).

En general, es de esperar que distintos áridos gruesos den lugar a distintas resistencias a la flexión en hormigones comparables. De ordinario las diferencias entre áridos son algo mayores en las propiedades que dan lugar a la resistencia a la flexión, que en aquellas otras que dan lugar a la resistencia a la compresión. Por ejemplo, Walker y Bloem, en su serie de ensayos antes citada (104), encontraron que la desviación máxima de la resistencia a la flexión para un árido dado, respecto de la media de todos los áridos, fue del 11 por 100. La desviación máxima encontrada por Kaplan en otra serie de ensayos (113) fue aproximadamente doble. 
Esta situación se complica aún más - ¿o tal vez se simplifica? - por el hecho de que las diferencias entre áridos respecto de las propiedades causantes de la resistencia a la flexión parecen depender también de la condición húmeda o no de la probeta de hormigón (128). A saber, estas diferencias tienden a desaparecer si se deja que el hormigón se seque.

\section{Hornmigón de alta resistemcia}

A causa del avance continuo en las estructuras ingenieriles, se cita cada vez con mayor frecuencia la necesidad de una producción práctica y rutinaria de hormigón de cemento portland de resistencia muy elevada. Por lo que respecta a la resistencia a la compresión, Mather ha preparado una revisión de la bibliografía relativa a hormigones con una resistencia a la compresión superior a las 10.000 libras/pulg. ${ }^{2}$ a los 90 días (129). Otros resultados han sido publicados por Smith y otros (130), en los que, entre otras cosas se discute también la importancia de los áridos minerales utilizados. Encontraron que tres de cada cinco áridos gruesos ensayados dieron lugar a resistencias a la compresión comparativamente altas en hormigones semejantes, mientras que los otros dos áridos fueron incapaces de proporcionar tan alto nivel de resistencia. Asimismo concluyeron que para hormigones de igual consistencia (asentamiento) y contenido de cemento, la arena natural producía mayores resistencias que las arenas artificiales. La ventaja se atribuyó a la menor exigencia de agua de amasado por parte del material menos angular.

Hay muchas más cuestiones que quedan por responder, en relación con el hormigón de elevada resistencia a la compresión. Esto es aún más cierto en el caso de hormigones de elevada resistencia a la flexión o a la tracción.

\section{Retraceión del hormigón}

La retracción de secado del hormigón es producida principalmente por la contracción del gel de silicato cálcico en la pasta de cemento endurecida, cuando el contenido de humedad de dicho gel disminuye (131). No obstante, hay además otros numerosos factores que influyen en la cuantía de la retracción. Un grupo de tales factores está relacionado con el árido mineral utilizado, debido a que al cambio de volumen de la pasta de cemento se opone en cierta medida el esqueleto constituido por el árido del hormigón. Los efectos del árido sobre la retracción del hormigón han sido estudiados por numerosos investigadores, tanto desde puntos de vista teóricos como experimentales. Algunas de estas investigaciones se discuten en lo que sigue.

Pickett (132), L'Hermite (133), Fulton (134), Hansen y Nielsen (135), etc., han desarrollado fórmulas teóricas o semiempíricas relativas al efecto que la cantidad de áridos minerales ejercen en la retracción. Por ejemplo, Pickett dedujo una fórmula para la reducción de la retracción total del hormigón causada por las partículas de árido, en los supuestos de que las partículas son esféricas y no retractivas, y que, además, tanto las partículas del árido como el resto del hormigón son elásticos. Esta fórmula es la siguiente:

en la cual:

$$
S=S_{0}(1-g)^{a}
$$

$$
\begin{aligned}
S= & \text { retracción del hormigón; } \\
S_{0}= & \text { retracción que tendría lugar si no existiera el árido; } \\
g= & \text { volumen de árido por unidad de volumen de hormigón, y } \\
a= & \text { factor que es una combinación de las constantes elásticas del árido y de la pasta } \\
& \text { endurecida, y que puede ser afectado por la fluencia de la pasta de cemento (136). }
\end{aligned}
$$


Experiencias llevadas a cabo para comprobar la validez de la ecuación [1] dieron resulta. dos bastante acordes con la fórmula.

Utilizando análogas hipótesis, Rostasy ha desarrollado un método para el cálculo de la retracción por secado y de las tensiones de retracción en el hormigón (137). Sobre esta base Wischers ha comprobado los efectos de varios factores en la retracción del hormigón (138). Sus resultados aportan la siguiente información concerniente a los efectos directos del árido sobre la retracción del hormigón.

a) cuanto menor es el tamaño de partícula mayor es la retracción que cabe esperar, supuesta fija la composición del hormigón. Sin embargo, el efecto del aumento del tamaño de partícula por encima de $1 / 2$ pulg. es despreciable;

b) cuanto mayor es el módulo de elasticidad del árido, tanto menor es la retracción que cabe esperar en el hormigón. No obstante, este efecto es también despreciable cuando el módulo de elasticidad es mayor que $5 \times 10^{6}$ libras/pulg. ${ }^{2}$, como es el caso corriente con áridos minerales normales y aceptables. Estos resultados obtenidos teóricamente están de acuerdo con las observaciones de Fulton (134).

Existe también una gran cantidad de material experimental disponible en la literatura técnica relativa al efecto de los áridos en la retracción del hormigón. Estos datos, en general, comprueban y completan los resultados teóricos. Por ejemplo, muchos datos experimentales demuestran que un aumento del contenido de pasta de cemento va acompañado de un aumento de retracción en hormigones comparables (139), (140) y (141), lo cual es otra forma de expresar la ecuación [1]. Igualmente, la retracción relativamente elevada de los hormigones con áridos ligeros (142) y (143), que constituye una de las propiedades más desfavorables de estos materiales, puede explicarse como resultado del bajo módulo de elasticidad de los áridos ligeros. Puesto que el módulo de elasticidad del agregado controla el módulo de elasticidad del hormigón endurecido (144) es de esperar que los hormigones con un alto módulo de elasticidad retraigan menos. Los resultados experimentales de Reichard (145) indican que tal correlación existe probablemente.

Los datos experimentales de Alexander y Wardlaw (146) demuestran claramente la influencia reductora de la retracción que ejerce el aumento del tamaño de las partículas, cuando las partículas son pequeñas. No obstante, además de este efecto directo del tamaño de las partículas hay otro aspecto relativo a la retracción que es muy importante en casos prácticos: el aumento del tamaño máximo de partículas, o la mejora de la granulometría, o ambas cosas, son eficaces en la disminución de la retracción, incluso indirectamente, debido a que permiten contenidos de agua menores (116 b), (139) y (147). Por ejemplo, Washa (131) informa de que un aumento del tamaño máximo del árido de $3 / 16$ a $3 / 4$ de pulg. disminuye la retracción aproximadamente en un tercio, en un hormigón con una relación agua/cemento y con una consistencia fijas. La opinión de Powers está de acuerdo con este resultado (148). Walker y Bloem (107) han observado una tendencia semejante, pero han notado también que en la zona de tamaños máximos comprendida entre $3 / 4$ y 2 1/2 pulg. las diferencias en las retracciones de secado eran muy pequeñas.

Como indica Meyers (149), varios investigadores han encontrado que una excesiva cantidad de arcilla u otro material fino de relleno en los áridos tiende a aumentar la retracción. Además de la cantidad, tiene importancia también la composición de la arcilla, como Hveem y Temper han demostrado (150). Para la evaluación, tanto de la cantidad como de la actividad de la arcilla contenida en los áridos, se recomienda el ensayo del equivalente de arena (117). La figura 5 muestra la buena correlación hallada entre los resultados de este ensayo 


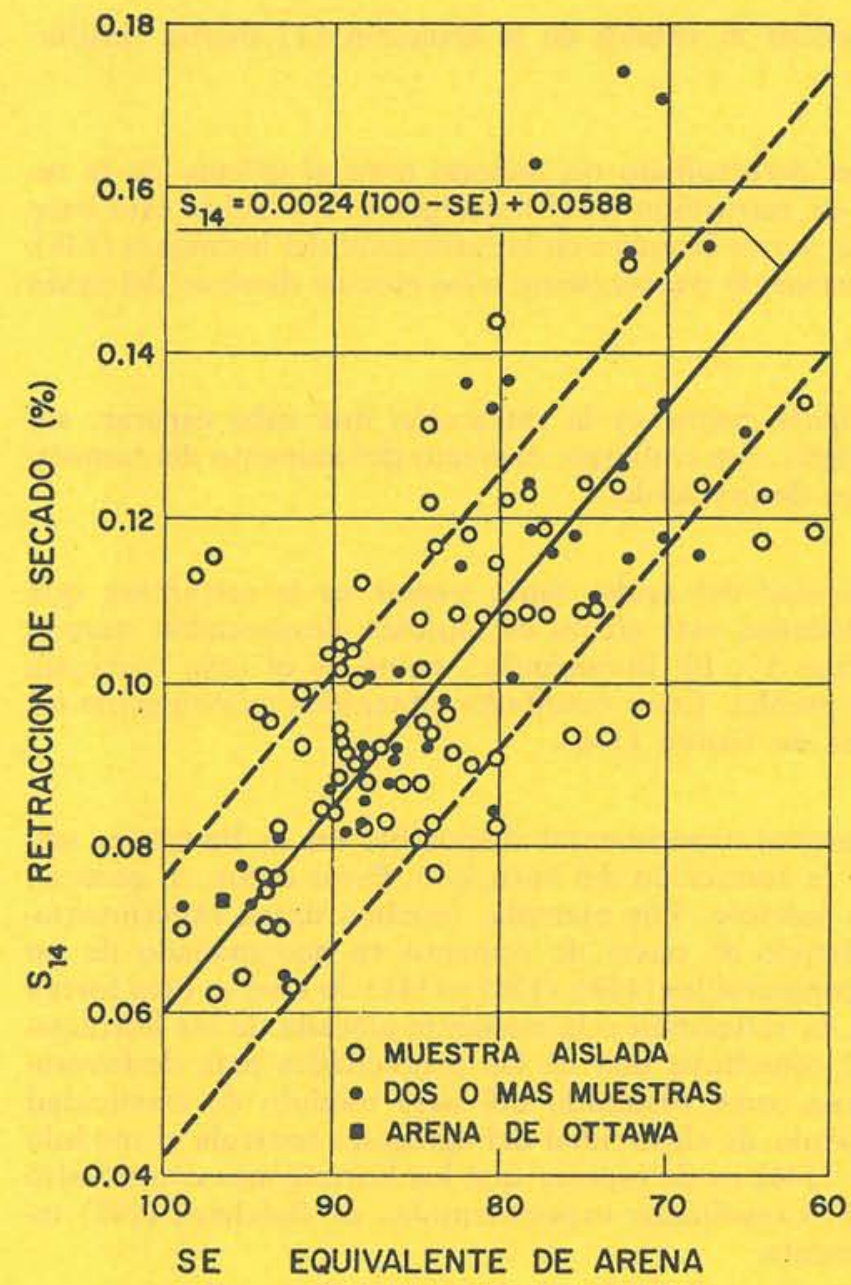

Fig. 5. Influencia del equivalente de arena en la retraceión de secado
del hormigón a los 14 dís (150).

Fig. 5. Influencia del equivalente de arena en la retracción de secado
del hormigón a los 14 días (150). fórmula es una modificación de la ecuación [1].

Finalmente, puede mencionarse que la fluencia o deformación del hormigón bajo carga está afectada por las mismas propiedades del árido que afectan a la retracción del hormigón.

Asimismo, es cierto que, en general, las propiedades de los áridos que tienden a aumentar la retracción tienden también a aumentar la fluencia del hormigón.

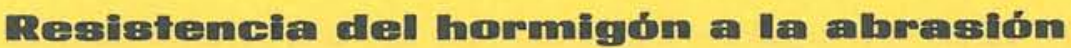

El desgaste abrasivo de las superficies de carreteras de hormigón producido por vehículos con neumáticos (sin cadenas) es generalmente insignificante, pero otra clase de vehículos pesados puede causar una considerable abrasión. Hasta ahora no existe en los Estados Unidos un método de ensayo normalizado para determinar la resistencia del hormigón a la abra- 
sión. Otros países han normalizado tales métodos, como por ejemplo el descrito en la norma alemana DIN 52108.

La abrasión de un hormigón está influenciada por numerosos factores. Un punto de partida lógico parece suponer que, puesto que la superficie del hormigón sometida a abrasión consta de pasta de cemento y de árido, la cuantía de la abrasión dependerá de la resistencia a la abrasión de ambos, pasta de cemento y áridos. La resistencia a la abrasión de la pasta de cemento depende de la relación agua/cemento, del contenido de aire y del curado, del mismo modo que la resistencia del hormigón depende de tales factores. Por lo tanto, y como numerosos resultados experimentales indican, existe una buena correlación entre ambas propiedades del hormigón, al menos hasta valores de resistencia a la compresión de 6.000 libras/pulg. ${ }^{2}$ Más allá de este límite los aumentos de la resistencia parecen ejercer poca influencia en la resistencia a la abrasión del hormigón. Kennedy y Prior (154), citando a Collins y Waters, indican que la velocidad inicial de desgaste de un hormigón de 2.000 libras/pulg. ${ }^{2}$ es aproximadamente cinco veces mayor que la de un hormigón de 4.000 libras/ pulgada cuadrada.

La influencia del tipo de árido mineral en la resistencia a la abrasión del hormigón, más allá y por encima de su contribución a la resistencia del mismo, es mucho menos clara. Smith, por ejemplo, extrajo las siguientes conclusiones de los resultados de sus ensayos hechos con 60 hormigones utilizando tres métodos distintos de abrasión (155):

a) la resistencia a la abrasión de un hormigón varía directamente con su resistencia a la compresión, con independencia de la calidad del árido o de las combinaciones de áridos utilizadas;

b) no se ha comprobado que exista una correlación significativa entre la calidad del árido grueso determinada, bien sea por el ensayo de estabilidad frente al sulfato sódico, o bien por el ensayo de abrasión Los Angeles, y la resistencia a la abrasión de los hormigones que contienen dicho árido.

Sin embargo, estas conclusiones tienen validez, estrictamente hablando, sólo para los áridos empleados por Smith en esta serie de ensayos; es decir, para áridos con un desgaste menor de 40 por 100 según el ensayo de abrasión Los Angeles, y con un tamaño máximo de $3 / 4$ de pulgada. El empleo de áridos más blandos puede rebajar la resistencia a la abrasión del hormigón. De acuerdo con los datos del Road Research Laboratory (114 c), si la resistencia a la compresión es igual o mayor que 6.000 libras/pulg. ${ }^{2}$, el tipo de árido ejerce poca influencia sobre la resistencia a la abrasión. Sin embargo, para hormigones de menor resistencia la influencia del tipo de árido puede ser considerable. Esto está comprobado por los datos de Graf relativos a la abrasión de probetas de hormigón seco (116 c). Graf halló igualmente que el efecto del tipo de árido es aún más pronunciado si el ensayo de abrasión se lleva a cabo con adición de agua. En tales circunstancias la abrasión normal (DIN 52 108) de un buen árido como cuarzo, basalto, pórfido, etc., es aproximadamente la mitad de la abrasión normal del hormigón hecho con el mismo árido (116 c).

La granulometría del árido también puede ejercer influencia en la resistencia a la abrasión del hormigón. Smith ha observado (115) que un aumento del porcentaje de arena en un hormigón dio por resultado un aumento de la abrasión cuando el árido era de buena calidad. Este hallazgo, que está de acuerdo con los datos de Price (156), puede explicarse mediante la hipótesis de que la buena resistencia a la abrasión de las partículas de árido grueso resulta más eficaz, con una reducción del contenido de árido fino. Además, datos experimentales de a'Court (157) indican que se produce un considerable aumento de la resistencia a la abrasión del hormigón al sustituir una arena fina por otra de buena granulometría (figura 6). Lo mismo demuestran los datos experimentales de Graf (116 c). La exudación del hormigón fresco puede afectar también a la resistencia a la abrasión. Puesto que la granulometría del hormigón es uno de los factores que controlan la exudación, también juzga un papel indirecto en ella. 


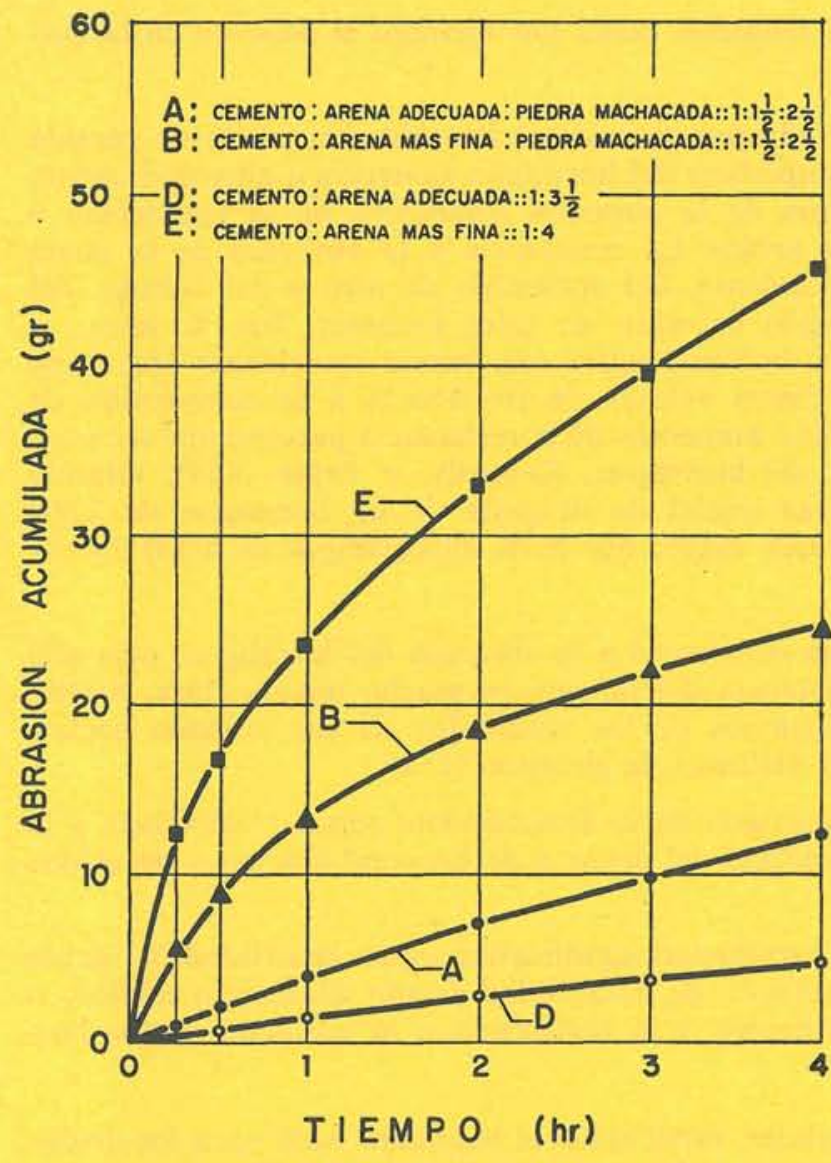

Fig. 6. Efecto de la granulometría del árido en la resistencia a la abra. sión del hormigón (157).
El efecto de la forma de las partículas en la resistencia a la abrasión del hormigón no ha sido investigado aún. No obstante, como indica $\mathrm{Li}$ (158), las formas angulares, aunque no laminares, de partículas son preferibles para una elevada resistencia a la abrasión.

Para ensayar la aceptabilidad de un árido para hormigón de pavimentación se recomiendan los métodos dados en las especificaciones AASHO y ASTM, además de los métodos descritos en las normas alemanas (159).

\section{Resis ctel hormefgón all clesilizammiember}

Hasta qué punto importa la resistencia al deslizamiento de los pavimentos de carretera desde el punto de vista de la seguridad lo demuestran las estadísticas corrientes que indican que el deslizamiento contribuye a más del 6 por 100 de todos los accidentes de tráfico en carretera. Aun cuando investigaciones recientes llaman la atención sobre el hecho de que el caucho utilizado en la fabricación de llantas, así como el diseño de las mismas, juegan un papel decisivo en el deslizamiento de los vehículos (160), generalmente se acepta que determinadas propiedades del árido mineral empleado en el hormigón ejercen una influencia significativa en la resistencia del pavimento al deslizamiento.

Se han desarrollado numerosos métodos de ensayo para medir la resistencia de un pavimento al deslizamiento (161) y (162). El conjunto de resultados de los ensayos muestra que las propiedades antideslizantes de la superficie de un pavimento bien proyectado y bien construido dependen en gran medida de las características de pulido del árido mineral con que el hormigón está hecho (163). Es de esperar que sea así, puesto que la superficie de un pavimento se pule continuamente a consecuencia del tráfico; así, cuanto mayor es la tendencia a pulirse el árido empleado, mayor será la reducción de la resistencia de la superficie al deslizamiento. Maclean y Shergold ensayaron la relación entre la magnitud del pulido de piedras en un ensayo de laboratorio (B.S. 812:1960) y la resistencia al deslizamiento de una carretera hecha con esquirlas de dichas piedras (164). Los resultados obtenidos con 28 piedras distintas muestran una buena correlación (fig. 7).

De acuerdo con experiencias anteriores, las superficies que contienen árido machacado tienen una mejor resistencia inicial al deslizamiento que las superficies hechas con áridos comparables redondeados. Sin embargo, después de un cierto período de uso la resistencia al deslizamiento de las dos superficies llega a ser prácticamente idéntica. 
En relación con la composición de la piedra, Shupe (163), señala que las calizas, como grupo, han alcanzado una pobre reputación con respecto a la susceptibilidad al pulido, si bien no todas las superficies de caliza resultan deslizantes, aun mojadas. Aquellas calizas que se pulen rápidamente poseen, bien sea una estructura cristalina de grano muy fino, o bien granos oolíticos redondeados contenidos en una matriz de calcita de dureza similar. Calizas como éstas no deben constituir la totalidad del árido superficial, porque tales superficies sólo de nuevas tienen una regular resistencia al deslizamiento; y pueden hacerse peligrosamente deslizantes después de haber soportado una moderada cantidad de tráfico. Las calizas que presentaron las mejores características antideslizantes fueron las granulares cristalinas compuestas de granos angulares entrecruzados y relativamente bajas en carbonato cálcico. La figura 8 presenta una relación empírica según Shupe (165) entre el contenido de carbonato cálcico de una caliza y su susceptibilidad al deslizamiento. La arenisca en una variedad de formas ha demostrado, de manera consistente, poseer excelentes características antideslizantes. Otras piedras con características de desgaste similares a las de la arenis-

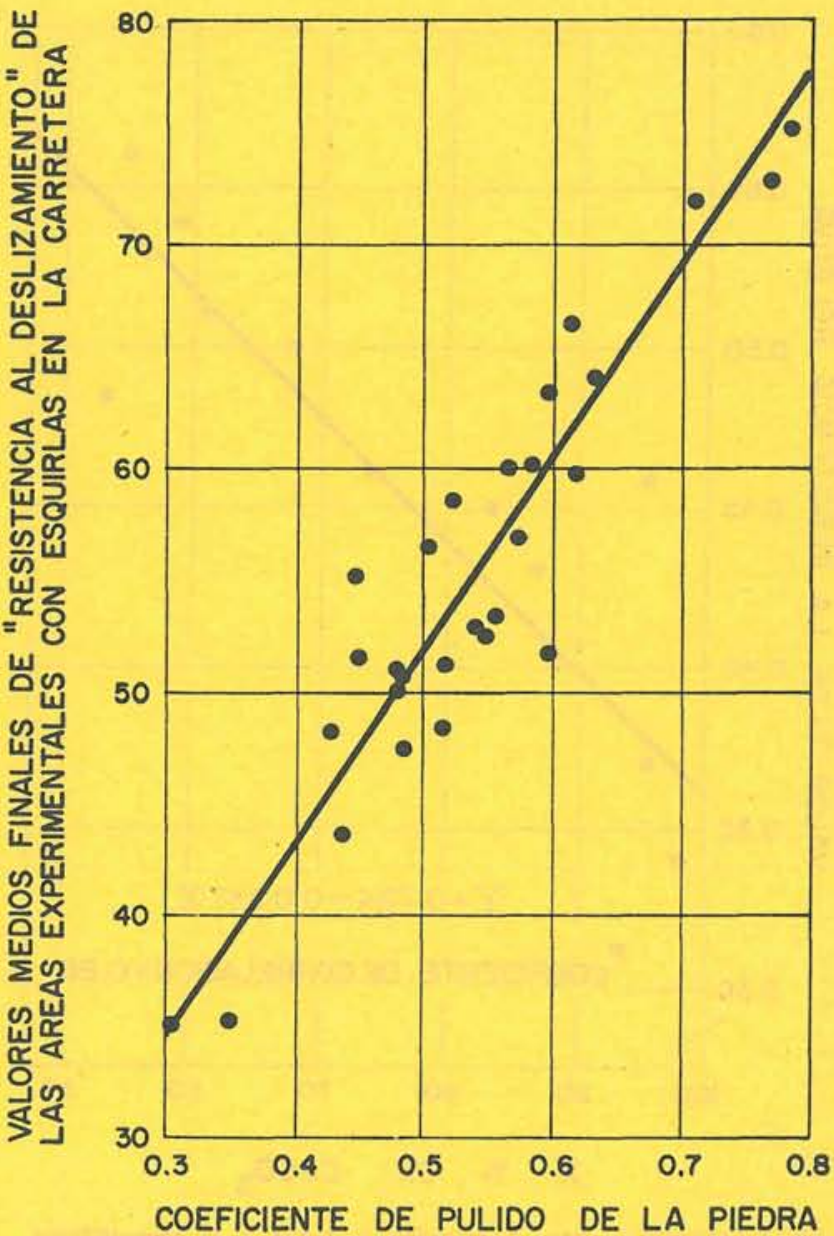

Fig. 7. Correlación entre los resultados del ensayo británico normal de puido de laboratorio y el estado final del pulido de esquirlas de una carretera recta muy transitada (164). $\mathrm{ca}$, tales como los granitos macrogranudos y las rocas trapeanas poseen también excelentes propiedades antideslizantes. Por el contrario, las piedras que tienen características de desgaste semejantes a las de la caliza pura, tales como el horsteno y la rhyolita, poseen escasas propiedades antideslizantes (163). Apoya esta opinión un informe de Shelburne y Dillard (166).

La buena resistencia del grupo de la arenisca al deslizamiento se atribuye a la estructura interna de la piedra. En general, las areniscas se componen de pequeños granos angulares de cuarzo conglomerados por una masa cementante más débil. Los granos individuales de cuarzo son altamente resistentes al pulido, puesto que el cuarzo ocupa el séptimo lugar en la escala de Mohs de durezas relativas. (La calcita, el componente principal de la caliza, ocupa el tercer puesto en dicha escala). Existe además en la arenisca el tipo de desgaste llamado "de partícula por partícula», lo cual significa que antes de que un grano de cuarzo expuesto al pulimento resulte altamente pulido, se separa de la masa cementante más débil, apareciendo en la superficie una nueva partícula dura (163).

Los pavimentos de hormigón, por regla general, no sufren este tipo de desgaste de partícula por partícula. No obstante, es posible producir un «desgaste diferencial». Este tipo de desgaste tiene la ventaja de que la resistencia del pavimento al deslizamiento seguirá siendo 


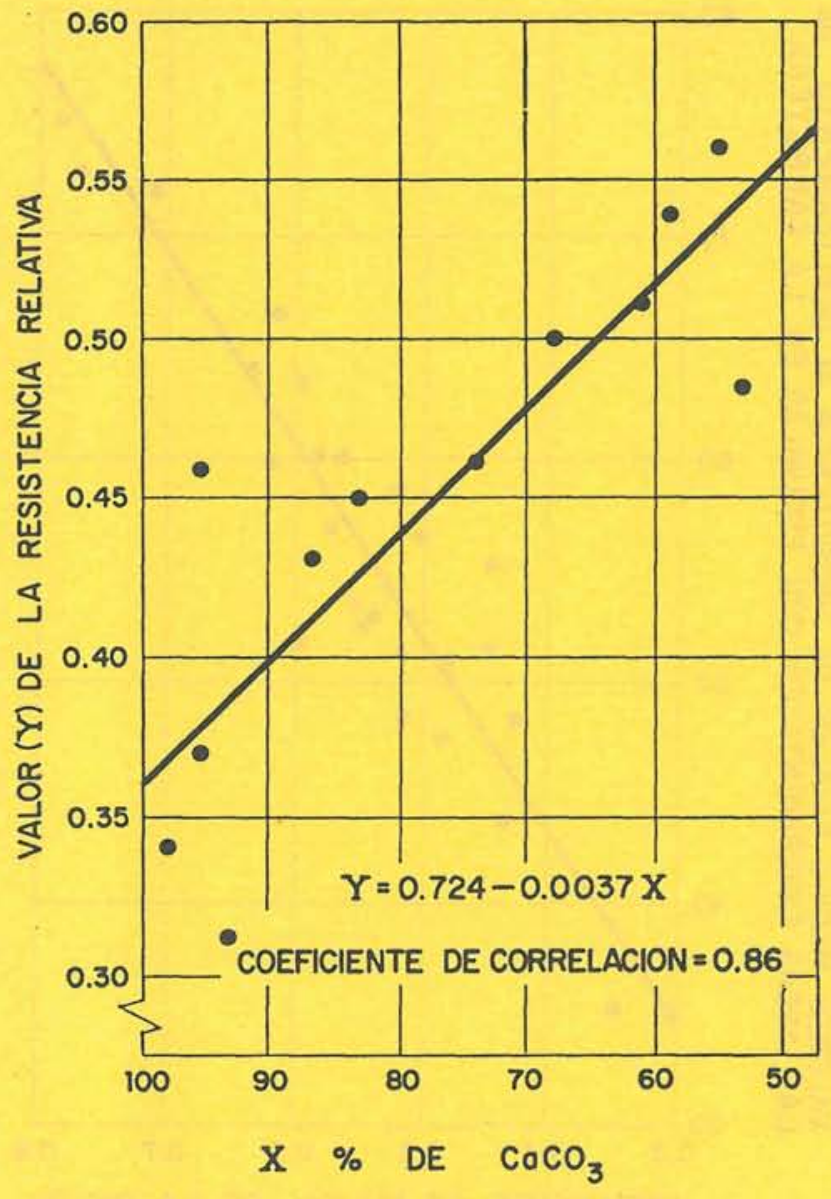
al deslizamiento de una caliza (165).
Fig. 8. Correlación entre el contenido de $\mathrm{CaCO}_{3}$ y la susceptibilidad

aceptable aun después de que el desgaste debido al tráfico haya progresado hasta el punto de quedar expuestas y al descubierto apreciables cantidades de árido grueso. El desgaste diferencial puede tener lugar a causa de variaciones de la resistencia al desgaste de la pasta de cemento y de los áridos finos y gruesos. Si el árido tiene una menor resistencia al desgaste que la pasta o el mortero cementante, lo cual es corriente cuando se trata de áridos calizos, las partículas se desgastan ligeramente por debajo de la superficie del mortero, aumentando así la rugosidad y la resistencia de la superficie al deslizamiento. Por otra parte, si el árido es el más resistente (grava de cuarzo), entonces las partículas quedan ligeramente por encima de la superficie del mortero, aumentando así también la resistencia al deslizamiento. En consecuencia, si en un pavimento de hormigón se utiliza un árido con características de pulido desfavorables, por ejemplo caliza, lo cual puede ocurrir frecuentemente por razones económicas, entonces debe utilizarse como árido fino arena cuarzosa natural, a fin de procurar un desgaste diferencial de la superficie del pavimento.

La resistencia al deslizamiento dependerá en menor medida de la granulometria del árido y del tipo de conglomerante; así, las conclusiones acerca de la resistencia al deslizamiento del hormigón de cemento portland se mantendrán también, más o menos, para la resistencia al deslizamiento de pavimentos asfálticos. Sin embargo, el árido requerirá normalmente un mayor esfuerzo de desgaste por pulido, y para llegar al último estado de resbalamiento, en el caso del hormigón de cemento portland que en el caso de superficies asfálticas (167).

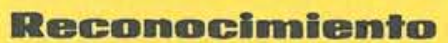

Este estudio fue particularmente sufragado por el State Highway Department de Alabama. El autor desea expresar su agradecimiento a este Departamento por la autorización para publicar este trabajo.

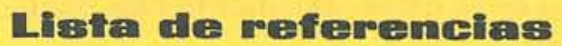

(101) SweEt, H. S.: «Physical and Chemical Tests of Mineral Aggregates and Their Significances». Symposium on Mineral Aggregates, ASTM STP, No, 83, Philadelphia, 1948.

(102) Woolf, D. O.: «The Relation between Los Angeles Abrasion Test Results and the Service Records of Coarse Aggregates», Proc. Highway Research Board, Vol. 17, p. 350, Washington, D. C., 1937. 
(103) Goldbeck, A. T.: «Discussion of the Los Angeles Abrasion Machine», Proc. ASTM, Vol. 35, Part. II, p. 530, 1935.

(104) WALKER, S., and BLOEM, D. L.: «Studies of Flexural Strength of Concrete», Part. I: «Effects of Different Gravals and Cements", NSGA, Circular No. 66, Washington, D. C., July 1956.

(105) Bloem, D. L., and Gaynor, R. D.: «Effects of Aggregate Properties on Strength of Concrete», ACI. Journal Proc., Vol. 60, No. 10, Oct. 1963.

(106) Walker, S., and Bloem, D. L.: Authors' Closure to Ref. (107), ACI Journal Proc., Vol. 57, March 1961.

(107) WALKeR, S., and BloEM, D. L.: «Effects of Aggregate Size on Properties of Concrete», ACI Journal Proc., Vol. 57, Sept. 1960.

(108) WalkeR, S.; BloEM, D. L., and GaynoR, R. D.: «Relationship of Concrete Strength for Maximum Size of Aggregate», Proc. Highway Research Board, Vol. 38, 1959.

(109) Higginson, E. C.; Wallace, G. B., and ORe, E. L.: «Effect of Maximum Size Aggregate on Compressive Strength of Mass Concrete", Symposium on Mass Concrete, ACI Publication SP-6, 1963.

(110) Corpon, W. A., and Gillespie, H. A.: "Variables in Concrete Aggregates and Portland Cement Paste which Influence the Strength of Concrete», ACI Journal Proc., Vol. 60, No. 8, August 1963.

(111) Popovics, S.: «Effect of Mineral Aggregate on Concrete Strength», Better Roads, September 1965.

(112) FrITSCH, J.: «Wovon hängt die Betonfestigkeit ab?» (Was Does the Strength of Concrete Depend on?), Zement-Kalk-Gips, H. 7, 1951.

(113) Kaplan, M. F.: «Flexural and Compressive Strength of Concrete as Affected by the Properties of Coarse Aggregates», ACI Journal Proc., Vol. 55, May 1959.

(114) RoAd RESEARCH LaboRatory: «Concrete Roads», Her Majesty's Stationery Office, London, 1955, p. 404. (a) pp. 66-67; (b) p. 133; (c) p. 79.

(115) U. S. Department of THE INTERIor BuREAU of Reclamation: Concrete Manual, Seventh Ed., U. S. Government Printing Office, Washington, D. C., 1963. (a) p. 103; (b) p. 61.

(116) Graf, O.; Albrecht, W., and Schaffler, H.: «Die Eigenschaften des Betons» (Properties of Concrete), Second Ed., Berlin-Göttingen-Heidelberg, Springer Verlag., 1960. (a) pp. 88-101; (b) pp. 242-245; (c) pp. 219-227.

(117) Hveem, F. N.: «Sand-Equivalent Test for Control of Materials During Construction», Proc. Highway Research Board, Vol. 32, 1953, p. 238.

(118) Newman, A. J., and Teychenne, D. C.: «A Classification of Natural Sands and its Use in Concrete Mix Design", Symposium on Mix Design and Quality Control of Concrete, London, May 1954, pp. 175$194+$ Discussion.

(119) SingH, B. G.: «Specific Surface of Aggregates Related to Compressive and Flexural Strength of Concrete», ACI Journal Proc., Vol. 54, No. 10, April 1958.

(120) ItakuRa, C.; Sugawara, T., and KaKu, T.: «Lean-Mix Rolled Concrete for Pavement Slabs», Proc. Highway Research Board, Vol. 37, 1958.

(121) LeNHARD, H.: «Zur Frage der praktischen Bedeutung der vollkommenen Frischbetonverdichtung» (Practical Significance of the Complete Compaction of Concrete), Zement, 31, Jahrg. (1942). Heft $11 / 12$ und Heft 13/14.

(122) Popovics, S.: "Consistency and its Prediction", RILEM Bulletin, No. 31, Paris, June 1966.

(123) Newman, K.: "The Effect of Water Absorption by Aggregates on the Water/Cement Ratio of Concrete», Magazine of Concrete Research, No. 33, Nov. 1959.

(124) KLIEGER, P.: «Early High Strength Concrete for Prestressing», Proc. World Conference on Prestressed Concrete, San Francisco, California, July 1957.

(125) GRIeb, W. E., and WeRner, G.: "Comparison of Splitting Tensile Strength of Concrete with Flexural and Compressive Strengths», Proc. ASTM, Vol. 62, pp. 972-990, 1962.

(126) Goldbeck, A. T.: «Concrete for Pavements», Crushed Stone Journal, Sept. 1946.

(127) Popovics, S.: «How to Improve the Flexural Strength of Concrete Made with Alabama Aggregates», Research Report, Auburn University, 1961.

(128) WALKER, S., and BLOEM, D. L.: «Studies of Flexural Strength of Concrete», Part. III: «Effects of Variations in Testing Procedure», Proc. ASTM, Vol. 57, 1957.

(129) Mather, B.: «Stronger Concrete». To be published by the Highway Research Board, Washington D. C. in 1967.

(130) SMITH, E. F.; TYNES, W. O., and SAUCIER, K. L.: «High-Compressive-Strength Concrete - Development of Concrete Mixtures», U. S. Army Engineer Waterways Experiment Station, Corps of Engineers, Miscellaneous Paper, No. 6-520, Report 2, Febr. 1964.

(131) Washa, G. W.: «Volume Changes and Creep», Significance of Test and Properties of Concrete and Concrete Aggregates, ASTM STP No. 169, Philadelphia, 1956.

(132) PICKETT, G.: "Effect of Aggregate on Shrinkage of Concrete and Hypothesis Concerning Shrinkage», ACI Journal, Vol. 52, p. 581, Jan. 1956.

(133) L'HeRmite, R.: Idees actuelles sur la technologie du béton (Timely Ideas in Concrete Technology), La Documentation Technique du Bâtiment et des Travaux Publics, p. 80, Paris, 1955. 
(134) Fulton, F. S.: «A Co-ordinated Approach to the Shrinkage Testing of Concretes and Mortars», Magazine of Concrete Research, No. 39, Vol. 13, Nov. 1961.

(135) Hansen, T. C., and Nielsen, K. E. C.: «Influence of Aggregate Properties on Concrete Shrinkage», ACI Journal Proc., Vol. 62, No. 7, pp. 781-794, July 1965.

(136) Neville, A. M.: Discussion of References (132), Part II, pp. 1380-81, ACI Journal, Dec. 1956.

(137) Rostasy, F. S.: "Zur Theorie der Austrocknung und des Schwindens zementgebundener Massen» (A Theory for the Drying and Shrinkage of Cement-Bound Masses), Zement-Kalk-Gips, 13, Jahrgang, Heft 3, Marz 1960.

(138) Wischers, G.: "Die mathematische Erfassung der Spannungen infolge Schwindens» (Calculation of Stresses caused by Shrinkage), Betontechniche Berichte, Beton-verlag, Dusseldorf, 1960.

(139) CarLson, R. W.: «Drying Shrinkage of Concrete as Affected by Many Factors», Proc. ASTM, Vol. 38, Part II, pp. 419-437, 1938.

(140) LYSE, I.: «The Shrinkage and Creep of Concrete», Magazine of Concrete Research, No. 33, 1959.

(141) LYSE, I.: «Fluage et Retrait du Béton» (Shrinkage and Creep of Concrete), RILEM Bulletin, No. 9 (New Series), pp. 93-99, Dec. 1960.

(142) SHIDELER, J. J.: "Lightweight Aggregate Concrete for Structural Use», ACI Journal Proc., Vol. 54, p. 299, Oct. 1957.

(143) Hummel, A.: Das Beton-ABC (The Alphabet of Concrete), 12th Ed., p. 28, Verlag von Wilhelm Ernst \& Sohn, Berlin, 1959.

(144) Hansen, T. C.: «Influence of Aggregate and Voids on Modulus of Elasticity of Concrete, Cement Mortar, and Cement Paste», ACI Journal Proc., Vol. 62, No. 2, pp. 193-216, Feb. 1965.

(145) ReIchard, T. W.: "Creep and Drying Shrinkage of Leightweight and Normal-weight Concretes», NBS Monograph, No. 74, National Bureau Standards, Washington, D. C., March 1964.

(146) AlEXANDER, K. M., and WardLAW, J.: "Effect of Powdered Minerals and Fine Aggregate on the Drying Shrinkage of Portland Cement Paste», ACI Journal Proc., Vol. 55, June 1959.

(147) Hummel, A.: "Vom Kriechen oder Fliessen des erhärteten Betons und seiner praktischen Bedeutung» (On Creep or Flow of Hardened Concrete and its Practical Significance), Zement, Vol. 24, No. 50 and 51, pp. 799-802 and 812-815, 1935 .

(148) Powers, T. C.: "Causes and Control of Volume Change», Journal of the PCA Research and Development Laboratories, Vol. 1, No. 1, pp. 29-39, Jan 1959.

(149) Meyers, B. L.: «A Review of Literature Pertaining to Creep and Shrinkage of Concrete», Engineering Bulletin Series, No. 56, Engineering Experiment Station, University of Missouri, Columbia, Missouri, Feb. 1963.

(150) HVEem, F. N., and TREMPER, B.: «Some Factors Influencing Shrinkage of Concrete Pavements», $A C I$ Journal, pp. 781-789, Feb. 1957.

(151) Troxell, G. E., and Davis, H. E.: Composition and Properties of Concrete, McGraw-Hill Book Company, Inc., New York-Toronto-London, 1956, pp. 236-239.

(152) L'Hermite, R.: «Volume Changes of Concrete», Chemistry of Cement, Proc. of the Fourth International Symposium, NBS Monograph 43, Vol. II, National Bureau of Standards, Washington, D. C., 1960.

(153) Snowdon, L. C., and Edwards, A. G.: "The Moisture Movement of Natural Aggregate and Its Effect on Concrete», Magazine of Concrete Research, No. 41, July 1962.

(154) Kennedy, H. L., and PRIOR, M. E.: «Abrasion Resistance», Significance of Test and Properties of Concrete and Concrete Aggregates, ASTM STP No. 169, Philadelphia, 1956.

(155) Smith, F. L.: "The Effect of Aggregate Quality on Resistance of Concrete to Abrasion", Cement and Concrete, ASTM STP No. 205, Jan. 1958.

(156) PrICE, W. H.: «Erosion of Concrete by Cavitation and Solids in Flowing Water», Journal of the American Concrete Institute, Vol. 19, No. 9, pp. 1009-1021, May 1947.

(157) A'Court, C. L.: "Mix Design and Abrasion Resistance of Concrete», Symposium on Mix Design and Quality Control of Concrete, Cement and Concrete Association, London, May 1954, pp. 77-91.

(158) LI, SHU-T'IEN: «Wear-Resistant Concrete Construction», ACI Journal Proc., Vol. 55, No. 8, pp. 879892, Feb. 1959.

(159) Strassenbau von A-Z (Highway Construction from A through Z), Erich Schmidt Verlag, Bielefeld, 1964.

(160) Moyer, R. A.: «California Skid Tests with Butyl Rubber Tires and Report of Visit to Road Research Laboratories in Europe Engaged in Skid Prevention Research", Highway Rsearch Record, No. 28, 1963.

(161) Anon.: First International Skid Prevention Conference, Part II, Virginia Council of Highway Investigation and Research, Charlottesville, August 1959.

(162) ASTM: Symposium on Skid Resistance, ASTM SPT No. 326, Dec. 1962.

(163) SHUPE, J. W.: «Pavement Slipperiness», Highway Engineering Handbook, Woods, K. B. (Ed.), Sec. 20, McGraw-Hill Book Company, Inc., 1960. 
(164) Maclean, D. J., and Shergold, F. A.: "The Polishing of Roadstones in Relation to Their Selection for Use in Road Surfacings», Journal of the Institution of Highway Engineers, Vol. 6, pp. 163-177, London, 1959.

(165) Shupe, J. W., and LounsBury, R. W.: "Polishing Characteristics of Universal Aggregates», Proc. of the First International Skid Prevention Conference, Virginia Council of Highway Investigation, Charlottesville, Virginia, 1959.

(166) Shelburne, T. E., and Dillard, J. H.: «An Appraisal of Skid Prevention», Proc. Ninth Pan American Highway Congress, May 1963, Chapter VI, Topic 15, Doc. No. 90, Organization of American States.

(167) Woops, K. B.; McLaughlin, J. H.: "The Role of Mineral Aggregates in the Design, Construction and Performance of Highway Pavements and Bridges", Proc. Ninth Pan American Higway Congress, May 1963, Chapter IV, Topic 10, Doc. No. 49, Organization of American States.

\section{mósummé summanty}

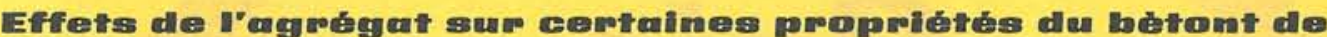

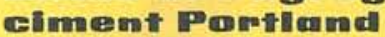

Sandor Popovics, professeur à l'Université d'Auburn, Alabama, U.S.A.

Dans ce travail est faite une description concise des effets que les propriétés principales des agrégats exercent sur le comportement du béton de ciment portland durci. Sont commentés les effets de la résistance, élasticité, composition chimique, grandeur et surface des agrégats. Les propriétés du béton durci objet de ces commentaires comprennent la résistance à la compression et flexion, contraction, résistance au frottement et au glissement.

\section{Effect of the cuggregate

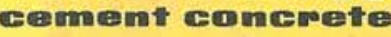

Sandor Popovics, Professor of Auburn University, Alabama, U.S.A.

This paper provides a concise description of the main effects due to the aggregate on the behaviour of hardened Portland cement concretes. The effects of strength, elasticity, chemical composition, size and surface of the aggregates are discussed. The properties of the hardened concrete which are taken into account are the compression and bending strength, surface wear and friction coefficient.

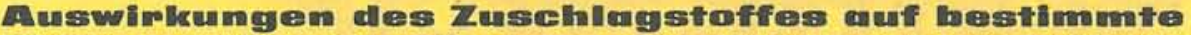

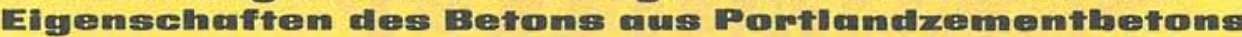

Sandor Popovics, Professor der Universität Auburn, Alabama, U.S.A.

Diese Arbeit gibt eine genaue Beschreibung der Auswirkungen, welche die Haupteigenschaften der $\mathrm{Zu}$ schlagstoffe auf das Verhalten des arhärteten Betons aus Portlandzement haben. Es werden die Effekte des Widerstandes, der Elastizität, der chemischen Zusammensetzung, der Grösse und Oberfläche der Zuschlagstoffe behandelt. Zu den hier besprochenen Eigenschaften des arhärteten Betons gehören der Widerstand bel Kompression und Flexion, sowie die Kontraktion und der Widerstand bei Reiben und Gleiten. 